\title{
Embolisation of pulmonary arteriovenous malformations - case series
}

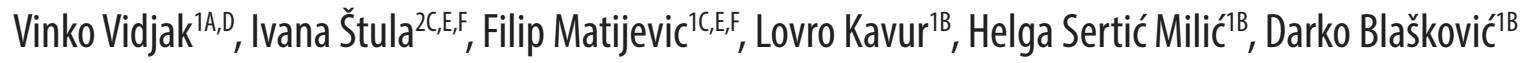 \\ 'Department for Diagnostic and Interventional Radiology, Clinical Hospital Merkur, Zagreb, Croatia \\ ${ }^{2}$ Department for Diagnostic and Interventional Radiology, Clinical Hospital Centre Split, Zagreb, Croatia
}

\section{Abstract}

Purpose: Pulmonary arteriovenous malformations (PAVM) are the direct communications between the pulmonary arteries and veins. These malformations can cause serious complications, and most of these patients should be treated. Herein we present our experience in the treatment of 18 cases of PAVM, treated with endovascular embolisation.

Material and methods: Eighteen patients with PAVMs underwent endovascular embolisation during a five-year period. Eight were male and 10 were female, with ages ranging from 16 to 65 years. Standard steel coils and vascular plug were used for embolisation.

Results: Embolisation was successful in 17 of 18 patients. Coiling was used in 10 patients, vascular plug in five, and both materials in two patients. All symptomatic patients with successful embolisation lost all their symptoms after treatment. Control angiography after embolisation showed a closure of AV shunt without migration of embolic material in all patients. Post-embolisation syndrome developed in four patients and late onset of pleural pain in three patients. There was no connection between pleural reaction and type of PAVM and embolic material.

Conclusions: Endovascular PAVM treatment is a minimally invasive, highly successful method with a low rate of only transitory complications.

Key words: pulmonary arteriovenous malformations, embolization, endovascular.

\section{Introduction}

Pulmonary arteriovenous malformations (PAVM) are rare lesions, caused by abnormal communications between the pulmonary arteries and veins, right-to-left shunt, causing decreased oxygenation of the arterial blood [1]. There are two types of PAVMs: simple and complex. Simple types (approx. 80\%) have a single feeding artery and a single draining vein, whereas complex types $(20 \%)$ have two or more of each [2,3]. Patients with PAVM can be asymptomatic for a long time, but they usually present with hypoxaemia and polycythaemia, mostly recognised by exercise intolerance and drumstick fingers [4]. These lesions are confirmed to be rarely sporadic, as it has been reported - between 60 and 90\% are congenital manifestations of autosomal dominant syndrome called hereditary haemorrhagic telangiectasia (HHT) [1,4-6]. Neurological events such as transient ischaemic attack (TIA), stroke, and cerebral abscess, the result of paradoxical embolisation, occur in $30-40 \%$ of patients with untreated PAVM [7-9]. These events are considered the main reasons for intervention. It is generally recommended that feeding arteries of $>3 \mathrm{~mm}$ be occluded to prevent paradoxical embolisation, even in asymptomatic patients [9-11]. The 3-mm threshold is a relatively arbitrary criterion, chosen when embolisation of smaller lesions was technically impractical. Currently, some interventional radiologists will treat all lesions identified on pulmonary angiography [12]. The conventional treatment of PAVM is surgical intervention, with all the complications that surgical procedure carries. That brings us to endovas-

Correspondence address:

Vinko Vidjak, Department for Diagnostic and Interventional Radiology, Clinical Hospital Merkur, 19 Zajčeva St., 10000, Zagreb, Croatia,

e-mail: vinko.vidjak@gmail.com

Authors' contribution:

A Study design · B Data collection · C Statistical analysis · D Data interpretation - E Manuscript preparation · F Literature search · G Funds collection 
cular embolisation, which we aim to present through our experience with 18 cases treated at our institution, with advantages over surgery such as a reliable therapeutic effect, a low complication rate, and reproducibility $[1,5,8]$.

\section{Material and methods}

During the last five years, 18 patients with PAVM had endovascular embolisation in the Clinical Hospital Merkur. The study was approved by the Ethics Committee, and each patient gave informed consent before inclusion in the study. Among them, eight (44.4\%) were male and 10 (55.6\%) were female, with ages ranging from 16 to 65 years with a median of 40 years (Table 1 ).

Indications for embolisation were diameter of the feeding artery lager than $3 \mathrm{~mm}$, lesion larger than $2 \mathrm{~cm}$, lesion with tendency for enlargement, and patients with neurological complications. Contraindications were respiratory tract infection, absolute contraindication for angiography, and pulmonary hypertension.

Only half of our patients were symptomatic. Three patients were presented with cerebral abscess, two with epistaxis, cyanosis was main physical sign in two cases, and exertional dyspnoea in two cases. In nine patients PAVMs were found during routine health examinations - by follow-up of previously reported chest X-ray abnormalities and dyspnoea with blood-saturation test abnormalities. HHT was associated in only two cases. No significant comorbidities were found in our patients.

Preoperative examination included laboratory examinations such as: routine blood, urine, liver and kidney function tests, blood coagulation tests, and blood oxygen saturation measurement. All patients had cardiological examination and chest X-ray. Enhanced chest multidetector computerised tomography (MDCT) scans were performed in 15 patients. Digital subtraction angiographys (DSAs) were performed before embolisation in three patients without MDCT scan in our institution, because they had had a recent scan performed at other institutions. Selective right and left pulmonary arteriography and super selective segmental arteriography were performed. Five patients had MDCT scan and DSA before intervention, due to better visualisation of feeding vessels.

Table 1. Presentation of all patients according to age, gender, symptoms, type of pulmonary arteriovenous malformations, embolisation material, and success of intervention with follow-up results during a period of one year

\begin{tabular}{|c|c|c|c|c|c|c|c|c|c|}
\hline Case & $\begin{array}{c}\text { Age } \\
\text { (years) }\end{array}$ & Gender & Symptoms & $\begin{array}{l}\text { Type and number } \\
\text { of PAVM }\end{array}$ & $\begin{array}{c}\text { Embolisation } \\
\text { material }\end{array}$ & $\begin{array}{l}\text { Technical } \\
\text { success }\end{array}$ & $\begin{array}{l}\text { Follow-up } \\
1 \text { months }\end{array}$ & $\begin{array}{l}\text { Follow-up } \\
6 \text { months }\end{array}$ & $\begin{array}{l}\text { Follow-up } \\
12 \text { months }\end{array}$ \\
\hline 1 & 53 & Female & HHT & 1 complex & Coils & Yes & Asymptomatic & Asymptomatic & Asymptomatic \\
\hline 2 & 38 & Male & Cyanosis & 1 simple & Coils & Yes & Asymptomatic & Asymptomatic & Asymptomatic \\
\hline 3 & 16 & Female & HHT & $\begin{array}{l}2 \text { complex, } \\
1 \text { simple }\end{array}$ & Coils & Yes & $\begin{array}{c}\text { Pleural pain } \\
\text { and effusion (MDCT) }\end{array}$ & Asymptomatic & Asymptomatic \\
\hline 4 & 29 & Female & None & 1 simple & Amplatzer & Yes & Asymptomatic & Asymptomatic & Missing \\
\hline 5 & 58 & Male & None & 1 simple & Amplatzer & Yes & Asymptomatic & Asymptomatic & Asymptomatic \\
\hline 6 & 40 & Male & Cyanosis & 1 complex & Amplatzer & Yes & Asymptomatic & $\begin{array}{l}\text { Pleural pain } \\
\text { (MDCT) }\end{array}$ & $\begin{array}{l}\text { Pleural pain } \\
\text { (MDCT) }\end{array}$ \\
\hline 7 & 38 & Female & None & 1 simple & Coils & Yes & Asymptomatic & Asymptomatic & Missing \\
\hline 8 & 32 & Female & None & 1 complex & Coils & Yes & Asymptomatic & Asymptomatic & Asymptomatic \\
\hline 9 & 27 & Male & None & 1 complex & Amplatzer + coils & Yes & Pleural pain (MDCT) & Asymptomatic & Asymptomatic \\
\hline 10 & 22 & Female & Abscess & 1 complex & Coils & Yes & Asymptomatic & Asymptomatic & Asymptomatic \\
\hline 11 & 39 & Female & Dyspnoea & 1 simple & Coils & Yes & $\begin{array}{l}\text { Pleural pain, } \\
\text { caugh (MDCT) }\end{array}$ & Asymptomatic & Asymptomatic \\
\hline 12 & 38 & Female & None & 1 simple & Coils & Yes & Asymptomatic & Missing & Missing \\
\hline 13 & 47 & Male & None & 1simple & Amplatzer + coils & Yes & Asymptomatic & Asymptomatic & Asymptomatic \\
\hline 14 & 49 & Male & None & 1 simple & Amplatzer & Yes & Asymptomatic & Asymptomatic & Asymptomatic \\
\hline 15 & 54 & Male & Dyspnoea & 1 complex & None & No & Missing & Missing & Missing \\
\hline 16 & 65 & Female & Abscess & 1 simple & Amplatzer & Yes & $\begin{array}{l}\text { Pleural pain, effusion, } \\
\text { temperature (MDCT) }\end{array}$ & Asymptomatic & $\begin{array}{l}\text { Pleural pain } \\
\text { (MDCT) }\end{array}$ \\
\hline 17 & 61 & Female & None & 1 complex & Coils & Yes & Asymptomatic & Asymptomatic & $\begin{array}{l}\text { Pleural pain } \\
\text { (MDCT) }\end{array}$ \\
\hline 18 & 59 & Male & Abscess & 1 simple & Coils & Yes & Asymptomatic & Asymptomatic & Asymptomatic \\
\hline
\end{tabular}

MDCT - multidetector computerised tomography, Coil - pushable coils 5-14 mm, Amplatzer - vascular plug, CVI - cerebrovascular incident, HHT - hereditary haemorrhagic telangiectasia 
During the procedure continuous monitoring of the electrocardiogram was done due to a risk of cardiac dysrhythmia. Also, blood pressure, respiration, and blood oxygen saturation were carried out during the intervention.

DSA and embolisation were made in all patients on a digital flat-panel angiography instrument (Shimatzu, Bransist Safire). A standard right femoral approach with an angled pigtail 5F (Terumo) for diagnostic right or left pulmonary arteriography was used in all cases. For a super selective segmental arteriography of feeding pulmonary arteries a different type of catheter was used. The lower lobe vessels, which were the most frequent site of PAVM, was catheterised by a curved catheter such as a Bernstein (Boston Scientific) catheter. In cases of difficult approach to the lesion, once a feeding artery was catheterised, a guiding $6 \mathrm{~F}$ catheter was placed in the parent segmental vessel to insure the stability of access and reduce the possibility of cardiac dysrhythmia. Oblique imaging was obtained from different angles to get an exact anatomy and size of the feeding artery, and in two cases we used 3D rotational angiography.

Heparin sodium was injected through a peripheral vein before embolisation. Percutaneous transcatheter embolisation was performed with two types of embolic material: coil and vascular plug. Embolic material was placed as close as possible to the venous part of the PAVM to reduce chances of recanalisation through the collateral circulation. We used pushable coils (Cook) with size from 5 to $14 \mathrm{~mm}$. The diameter of the coil was equal to or $10 \%$ larger than the diameter of the feeding artery (Figure 1). Two techniques of embolisation were used: "anchor" and "scaffold", to reduce the risk of coli migration. In the anchoring technique the coil tip is anchored in a small side-branch proximal to the actual malformation, and the body of the coil then prolapses into the feeding vessel. In the scaffold technique a larger coil is used initially to create a scaffold into which smaller coils can be placed to build up the coil nest. In patients with complicated vascular anatomy, such as large or short feeding artery, an Amplatzer vascular plug II (St. Jude Medical, AGA Medical Corporation) was used because the screw mechanism of release allows precise deployment and reposition (Figure 2).

The follow-up intervals of one, six and, 12 months included clinical examination, routine blood tests, blood oxygen saturation, and chest X-ray in all 17 patients. In the case of symptom appearance in the follow-up period, coil migration reported on chest X-ray, or abnormal oxygen saturation test, contrast-enhanced thorax MDCT was planned. Because all of our patients already required long-term monitoring, we decided that the radiation risk of multiple MDCTs during the follow-up period was too high, which is why we did not perform MDCT follow-up in any of our patients.

The data were analysed using descriptive test, chisquare test; Statistica for Windows Release 7.0 Statsoft,
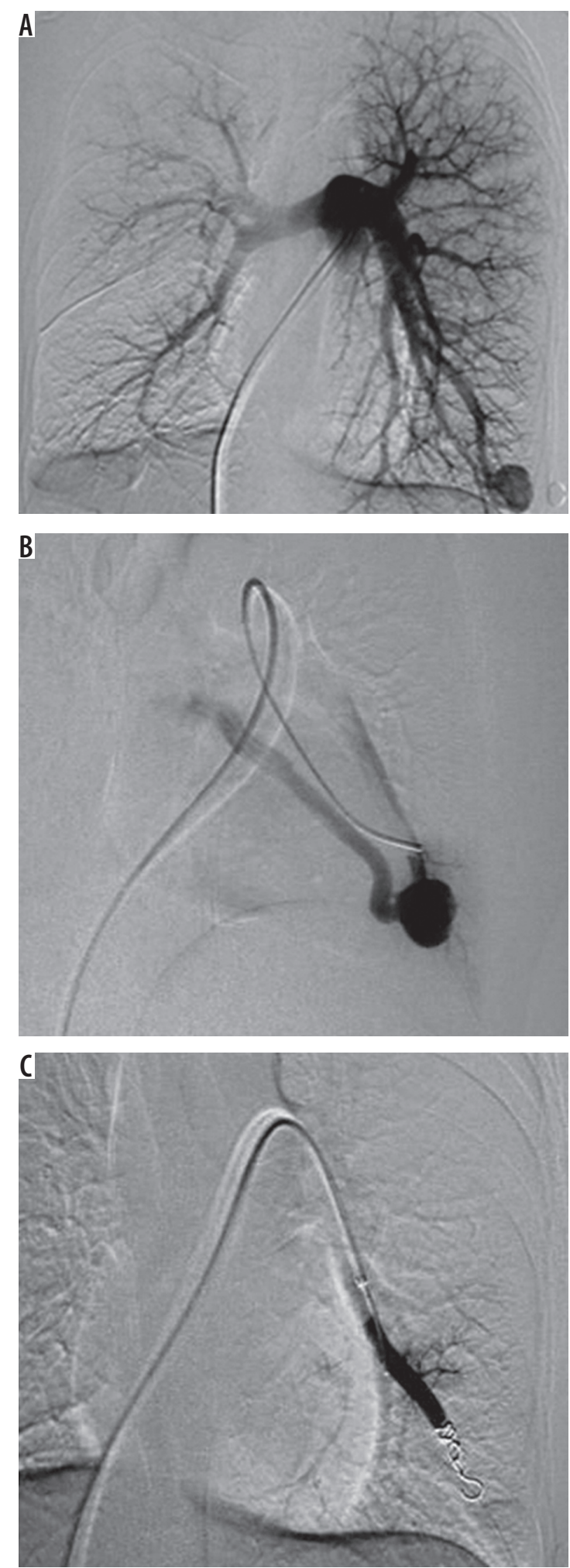

Figure 1. Colling of the left lower lobe simple pulmonary arteriovenous malformations

Tulsa, Oklahoma, USA. All $p$ values less than 0.05 were considered to indicate statistical significance.

\section{Results}

Simple PAVMs were found in 10 patients (56\%) and complex lesion with blood supply of the lesion from more than 

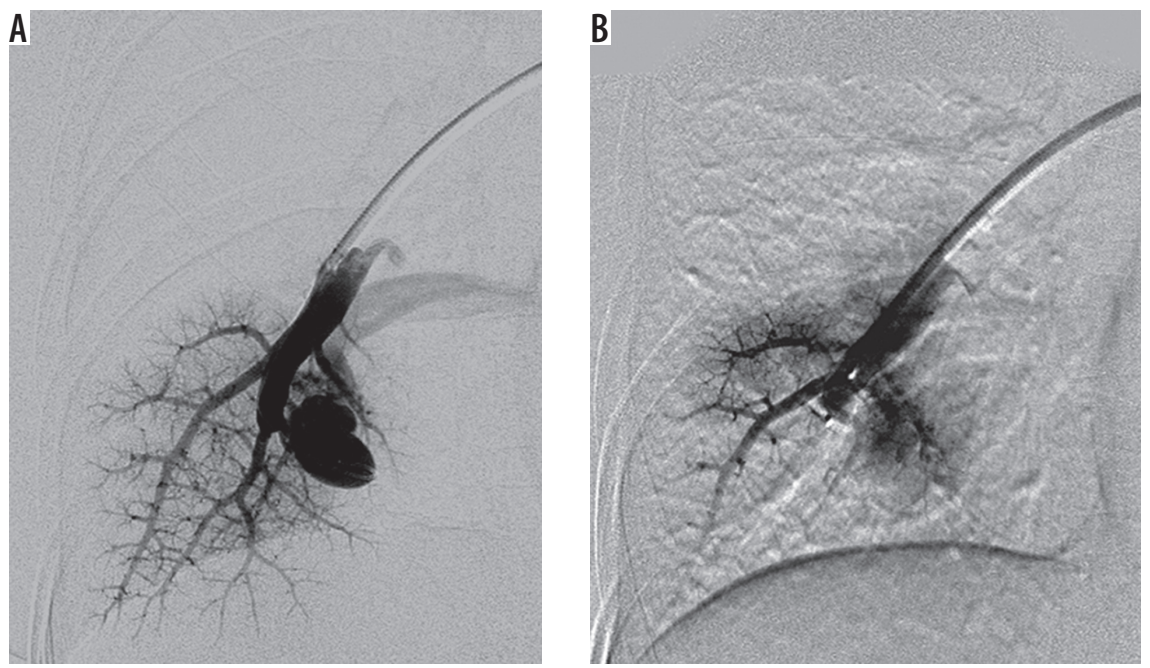

Figure 2. Embolisation of the right lower lobe simple pulmonary arteriovenous malformations with vascular plug

two segmental arteries or veins in eight patients (44\%). Single PAVM lesions were found in all cases except one who had three lesions: one simple and two with more than two feeding arteries (Table 1).

We managed to perform successful embolisation in all patients except one who had extreme tortuosity of parent vessels, so it was impossible to make a safe embolisation. The coils were used in 10 (58.8\%) patients (Figure 1). Amplatzer vascular plug was used in five (29.4\%) patients (Figure 2). In two (11.8\%) patients both vascular plug and coils were used. One of them had two feeding arteries; one was closed with coils and another with an Amplatzer plug. In a second patient, after the feeding artery had been closed with a vascular plug, control angiography showed a small feeding artery, which was closed with coils. In all patients control pulmonary angiography after embolisation was performed (Table 1).

Control angiography after embolisation showed in all patients a closure of AV shunt without migration of embolic material (Figure 3). A normal vascular anatomy nearby the lesion was preserved.

All symptomatic patients with successful embolisation lost all their symptoms after treatment. Post-embolisation syndrome was present in four (23.5\%) patients. In all four patients mild chest pain occurred a few days after the embolisation with small pleural effusion in two (11.8\%) patients. One of the patients with pleural effusion had also a high temperature. A cough was present in one patient with pleural pain. The late onset of pleural pain occurred in three $(17.6 \%)$ patients. Pleural pain started four months after embolisation in one patient, and in two patients after one year. One of the patients with late onset of pleural symptoms had also pleural effusion in the early post-embolisation period (Table 1).

Contrast enhanced thorax MDCT was done in six (35.2\%) patients with a pleural reaction symptom (Table 1). In four patients, MDCT was made one month after embolisation, in one after six months, and in three patients one year after embolisation. Two of these patients had
MDCT twice. One of them had pleural effusion in the early post-embolisation period and late onset of pleural pain after one year, and another had MDCT six months and one year after embolisation due to pleural pain (Table 1). Pleural effusion was found on thorax MDCT in two patients with pleural pain in the early post-embolisation period (follow-up one month). There was no pathological change on thorax MDCT in patients who developed symptoms in the late post-embolisation period. In most patients the post-embolisation symptoms disappeared in a short period, regarding only symptomatic treatment. Only one patient with pleural effusion and a high temperature received antibiotic therapy.

After the embolisation blood oxygen saturation increased to normal levels in all patients without signs of hypoxemia. In all patients, the follow-up chest X-ray showed either complete disappearance of PAVM or its replacement with fibrous structure. The embolic material stayed in the same position during follow-up.

There were no significant differences between simple and complex PAVM lesions regarding embolic material $\left(\chi^{2}=1.31, p=0.52\right)$ and clinical outcome $\left(\chi^{2}=1.32\right.$, $p=0.25)$. Post-embolisation syndrome did not show connection with type of PAVM $\left(\chi^{2}=0.42, p=0.52\right)$, embolic material $\left(\chi^{2}=0.88, p=0.64\right)$, or symptomatic PAVM $\left(\chi^{2}=1.29, p=0.26\right)$. The same was true with the late onset pleural pain that was not connected with embolic material $\left(\chi^{2}=2.86, p=0.24\right)$, type of PAVM $\left(\chi^{2}=0.42, p=0.51\right)$, and symptomatic PAVM $\left(\chi^{2}=0.14, p=0.71\right)$.

\section{Discussion}

Pulmonary arteriovenous malformations (PAVMs) are direct communications between pulmonary arteries and veins. It is a rare disorder with the incidence of 2-3 per 100,000 population [13]. PAVMs are usually congenital malformations but rarely may be acquired. It is more frequent in females, and the male-to-female ratio varies from $1: 1.5$ to $1: 1.8$ [14]. In our study, female gender was 

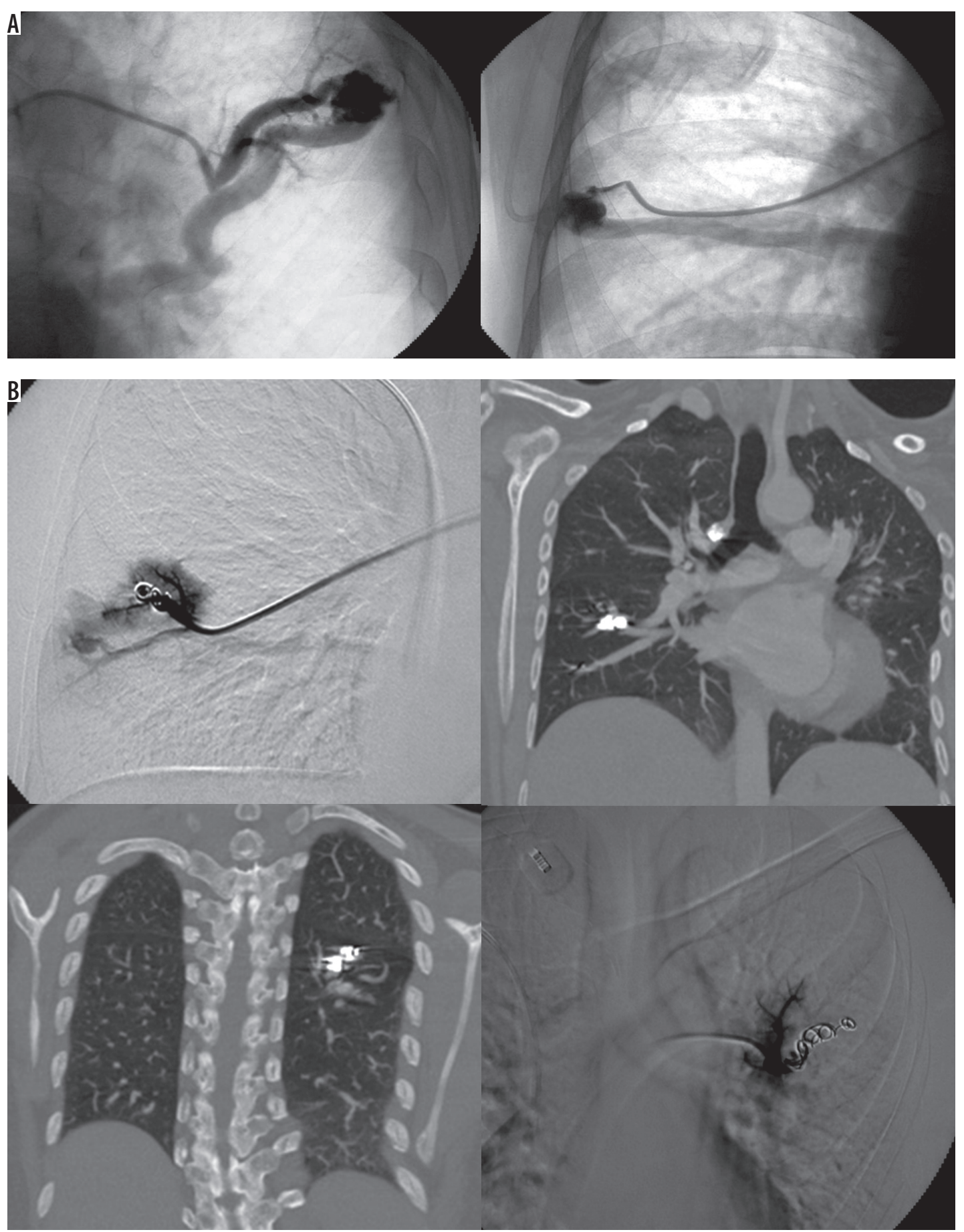

Figure 3. A) Angiography before embolisation. B) Digital subtraction angiography and maximum intensity projection reconstruction of contrast enhanced thorax multidetector computed tomography after pulmonary arteriovenous malformations embolisation

more frequent also, but the ratio was not very high. Mostly PAVM were simple, consisting of a single feeding artery and single draining vein, as is usual in cases of PAVM. The proportion of complex type PAVM, which has two or more feeding arteries or drainage veins, was $44 \%$ in our patients, which is higher than in most published studies $[1-5,13]$. The type of PAVM did not have any influence on the embolisation outcome. Most PAVMs were single and localised to the lower lobes, as is usual for PAVM.

PAVMs caused right-to-left shunt, which, if small, will be clinically silent. In different studies asymptomatic patients account for between 13 and 55\% of all patients [14-16]. Almost half of our patients were asymptomatic. Symptoms develope when right-to-left shunt exceeds $25 \%$ of total blood volume. Decreased blood oxygenation caused symptoms of hypoxaemia such as cyanosis and dyspnoea, as we saw in some of our patients. If high shunt flow is present, it will result in an increased cardiac output and heart failure at the end, which did not occur in our study.

PAVM should be considered in differential diagnosis of young patients with cerebral abscess, TIA, or stroke. PAVM also should be excluded in patients with unex- 
plained hypoxia or cyanosis, and in first-degree family members with HHT. Hereditary haemorrhagic telangiectasia (HHT) is an inherited disorder of the vasculature associated with arterio-venous malformation and telangiectasias. Approximately $70 \%$ of PAVM cases are associated with HHT, but only $25-30 \%$ of patients with HHT have pulmonary AVM due to different genetic mutations responsible for the disease $[17,18]$. In our study we had only two patients with HHT; the diagnosis of HHT was made by clinical symptoms known as Curacao criteria (epistaxis, mucocutaneous telangiectasia, and PAVM) [19].

Severe cerebrovascular incidents such as TIA, stroke, and cerebral abscess occur in 30-40\% patients [7-9]. The mechanisms are mostly paradoxical embolism from systemic thrombus, but also lack of filtration and increased blood viscosity can cause cerebral accidents. Although cerebral abscess is an initial symptom of PAVM, in 5-14\% of patients in our study it was the main cerebral event $[13,20]$. A possible explanation for this is a non-sterile thrombus from the vein due to thrombophlebitis in two patients. In one patient the probable explanation was earlier pulmonary infection near the vascular malformation.

These malformations can caused serious complications, and there is an evidence-based indication to treat these patients. In untreated patients, according to published data, morbidity is the range $26-33 \%$ and mortality from 8 to $16 \%$ [18]. The risk for central nervous system complications is significant in all PAMs with a feeding artery $\geq 3 \mathrm{~mm}$, so this characteristic of the lesion demanded treatment regardless of symptoms [9-11,17].

Chest X-ray is not an adequate diagnostic tool for PAVM because some PAVMs are not visible on chest $\mathrm{X}$-ray, depending on the size and location. We made MSCT angiography or DSA in all our patients before intervention because these methods are highly sensitive in PAVM diagnostics and can precisely show afferent and efferent vessels [21-23]. In the same case we used both methods to make an exact visualisation of the feeding vessel, and in two patients 3D rotational angiography was used. Exact measurement of the feeding vessel is necessary to choose the right embolisation material that prevents a paradoxical embolisation or PAVM recanalisation.

Today, embolisation is the method of choice in the treatment of PAVM, due to its high success rate and only a few complications $[1,5,11,24]$. Also, embolisation is a minimally invasive procedure, which requires only local anaesthesia and significantly shorter time of hospitalisation compared to surgical treatment. Technical success of embolisation with occlusion of the feeding artery can be achieved in $95-100 \%$ of cases. We managed to occlude the feeding artery in all cases except one patient in whom it was impossible to safely catheterise the feeding vessels.

Recanalization is always is possible from adjacent pulmonary arteries. Recanalisation after use of coils has been described in $8-16 \%$ of cases $[25,26]$. There were no recanalisations in our patients during the 12 -month control period. We always used the "anchor" or "scaffold" technique, which reduced the recanalisation rate. Failure of endovascular treatment of PAVM occurs mostly with large and short feeding vessels, due to catheter instability during the procedure and high risk of coil migration. Surgery is a safe option for large, single, and centrally located lesions [27]. Currently, new techniques such video-assisted thoracoscopy are being used.

The most serious complication of endovascular treatment is paradoxical embolisation, with an incidence of $0.5-2.5 \%[13,28,29]$. Paradoxical embolisation occurred due to an overly large orifice of the malformed vessel, short neck of the feeding artery, small diameter of embolic material, or high blood flow rate. Exact measurement of the feeding vessel size and appropriate embolisation material is crucial to avoid this complication. In the case of difficult anatomy we used a few selective angiograms of the PAVM from different angles, to make the most precise measurement of the feeding artery. The size of the embolic material was $\geq 10 \%$ larger than the size of the feeding artery in our study. Much larger size of embolic material would be absolute insurance against paradoxical embolisation, but it would be impossible to perform correct curling of the coil inside the feeding artery and its complete occlusion. Coils are flexible, and they are preferred in tortuous and small calibrated vessels. For short and large feeding vessels we used an Amplatzer device [30,31]. It is a selfexpandable cylindrical nitinol wire-mesh occlusion device, which is detachable and can be repositioned very precisely with safe controlled detachment. We manage the problem of fast blood flow also using an Amplatzer vascular plug. In the case of fast blood flow, a temporary occlusion of the proximal part of the feeding vessel can be achieved by using a balloon catheter. Another solution in the case of short and large feeding vessel or fast flow could be the use of a covered stent [1]. We did notice migration of embolic material in or patients during procedure and in flow-up period.

Post-embolisation syndrome is result of a body reaction to tissue infraction. As part of post-embolisation syndrome, slight to moderate chest pain and transient lowgrade fever occurred in $10-15 \%$ of patients $[4,9,12]$. Pleural reaction usually starts in the first few days after embolisation. It is self-limited and usually stops during the subsequent 4-7 days. In our study four patients had post-embolisation syndrome in the first week after embolisation, and two of them developed pleural effusion. One patient who had a high fever was treated with antibiotics and another needed only short pain-relief therapy. Unusually, we had one patient who developed pleural pain after four months, and three patients one year after embolisation. In cases of pleural symptoms with routine chest X-ray, patients had also thorax MDCT. MDCT was normal in all symptomatic patients except in two of them, who had pleural effusion in the early post-embolisation period. We did not find a connection between the pleural reaction and the type of the PAVM or embolic material. An Amplatzer device was 
more frequently used in patients with late onset of pleural symptoms but without significant difference.

All patients with successful embolisation experience experienced disappearance of their symptoms with higher functional level.

\section{Conclusions}

Embolisation should be the first choice in PAVM treatment because it is a minimally invasive, highly successful method with a low rate of only transitory complications. Development of a new embolic material such as vascular plug makes embolisation possible in almost all patients independently of vascular anatomy.

\section{Conflict of interest}

The authors report no conflict of interest.

18. Swanson KL, Prakash UBS, Stanson AW. Pulmonary arteriovenous fistulas. Mayo Clin Proc 1999; 74: 671-680.

19. Shovilin CL, Guttmacher AE, Buscarini E, et al. Diagnostic criteria for hereditary haemorrhagic telangiectasia (Rendu-Osler-Weber syndrome). Am J Med Genet 2000; 91: 66-67.

20. Rao SC, Main ML. Transesohageal echocardiographic diagnosis of pulmonary arteriovenous malformation in a patient with ischaemic stroke. Eur J Echocardiogr 2009; 10: 347-349.

21. Nawaz A, Litt HI, Stavropoulos SW, et al. Digital subtraction pulmonary arteriography versus multidetector CT in the detection of pulmonary arteriovenous malformation. J Vasc Interv Radiol 2008; 19: 1582-1588.

22. Tsunezuka Y, Sato H, Tsukioka T. Strategy for 3-D computed tomography diagnosis and treatment of small pulmonary arteriovenous fistula. Scand Cardiovasc J 2000; 34: 90-91.

23. Remy J, Remy-Jardin M, Giravd F, Wattinne L. Angioarchitecture of pulmonary arteriovenous malformation: clinical utilities of three-dimensional helical CT. Radiology 1994; 191: 657-664.

24. Gupta P, Mordin C, Curtis J, et al. Pulmonary arteriovenous malformation effect of embolisation on right-to-left shunt, hypoxemia and exercise tolerance in 66 patients. AJR Am J Roentgenol 2002; 179: 347-355.

25. Andersen PE, Kjeldsen AD. Clinical and radiological long-term follow-up after embolisation of pulmonary arteriovenous malformation. Cardiovasc Intervent Radiol 2006; 29: 70-74.

26. Lee DW, White RI Jr, Egglin TK, et al. Embolotherapy of large pulmonary arteriovenous malformations;long-term results. Ann Thorac Surg 1997; 64: 930-940.

27. Georghiou GP, Berman M, Vidne BA, Saute M. Pulmonary arteriovenous malformation treated by lobectomy. Eur J Cardiothorac Surg 2003; 24: 328-330.

28. Prasad V, Chan RP, Faughnan ME. Embolotherapy of pulmonary arteriovenous malformations: efficacy of platinum versus stainless steel coils. J Vasc Interv Radiol 2004; 15: 153-160.

29. Grosso M, Groppo Marchisio F, Testa F, et al. Pulmonary arteriovenous malformations: percutaneous treatment preserving parenchyma in high-flow fistulae. Radiol Med 2008; 113: 395-413.

30. Wang W, Li H, Tam MD, et al. The amplatzer vascular plug. A review of the device and its clinical applications. Cardiovasc Intervent Radiol 2012; 35: 725-740.

31. Hart JL, Aldin Z, Braude P, et al. Embolisation of pulmonary arteriovenous malformations using the Amplatzer vascular plug: Successful treatment of 69 consecutive patients. Eur Radiol 2010; 20: 2663-2670. 